\title{
Complex trapezoid grating for light trapping in thin-film solar cells: super-fine structure
}

\author{
Junfei FANG ${ }^{1,2}$, YuChun GOU ${ }^{1,2^{*}}$, JiANPING DENG $^{1,2}$ \\ ${ }^{1}$ Shaanxi Key Laboratory of Industrial Automation, Shaanxi University of Technology, \\ Hanzhong 723001, China \\ ${ }^{2}$ School of Mechanical Engineering, Shaanxi University of Technology, \\ Hanzhong 723001, China
}

${ }^{*}$ Corresponding author: ycgou@snut.edu.cn

\begin{abstract}
The research of the optimal surface structure has attracted considerable interest because of its potential application in light trapping in thin-film solar cells (TFSCs). In this paper, a super-fine structure named complex trapezoid grating is proposed to improve the optical absorption comparing to the conventional simple trapezoid grating in a-Si:H TFSCs. The numerical calculation by utilizing rigorous coupled-wave analysis (RCWA) is conducted to obtain the optical absorption of the structured surface. The results demonstrate that, compared to a planar slab, the optimized-simple trapezoid grating shows $97 \%$ enhancement of power conversion efficiency $\eta$ while the complex trapezoid grating shows $131 \%$ enhancement. Obviously, the complex trapezoid grating exhibits a better performance than the simple grating, which is due to the perfect antireflective effect and microcavity resonance effect. The angular response of the optical absorption in a-Si:H TFSCs was also investigated. The results further indicate that it is a better way to select the complex trapezoid grating in improving the optical absorption of silicon-based TFSCs.
\end{abstract}

Keywords: light trapping, complex trapezoid grating, rigorous coupled-wave analysis, solar cells.

\section{Introduction}

Solar cells technology is considered to be the most promising approach in future energy system because of the fossil energy shortage and environmental pollution. Silicon -based TFSCs have attracted significant interest as the candidates for future generation of photovoltaics due to their mature fabrication technology, less consumption of material and low cost. In addition, compared with the bulk solar cells, the other obvious advantages of TFSCs are the effective carrier extraction and reductive carrier-hole recombination, which leads to lower dark current, higher open-circuit voltage $V_{\text {oc }}$ and higher fill-factor (FF) [1]. Unfortunately, a primarily unavoidable drawback of silicon -based TFSCs is poor optical absorption due to its lower optical absorption coefficients, which limits the conversion efficiencies of the cells. Therefore, in order to overcome 
this limitation and to improve the optical absorption in TFSCs, light trapping techniques were widely used and became exceptionally essential in this realm.

Light trapping generally refers to the approaches for redistributing the incoming light into new directions within the solar cells [2] ]. Its purpose is to prevent this redirect light from escaping the solar cells and improve the optical absorption within the active layer as far as possible. So it consists of two major aspects: one is to reduce the reflection of the front surface and the other is to increase the optical path length for light travelling in the active layer. Up to date, much effort has been devoted to design light trapping structures in the realm of TFSCs. Various novel structures have been proposed. The typical representations among these novel structures are metallic nanoparticles [3-7] and periodic metallic gratings [ $\underline{8}-\underline{11}]$. For these structures, metal plays an important role to strongly localize the electromagnetic field within the cells, which is the consequence of exciting the plasmon polaritons. However, the enhanced electromagnetic field usually surrounded the metallic nanostructures. Metal has strong absorption ability due to its high optical absorption coefficients. It is well-known that the light was absorbed by two parts of metallic nanostructures and the active layer. Therefore, the introduction of metallic nanostructures has the tendency to increase parasitic optical absorption of the metal, which leads to the inefficient use of the incident solar spectrum and even decreases the absorption in the active layer [12]. It should be noted that the incident energy only absorbed by the active layer can generate the electron-hole pairs, or else it will become heat in metal, which is useless. Although the total absorption is highly enhanced by using metallic nanostructures, the absorption in the active region is not evidently enhanced.

Recently, a promising approach in designing the structure of solar cells is to pattern the active layer itself as a grating or photonic crystals [13-16]. Such nanophotonic structures can excite waveguide mode or leaky-mode resonances inside the active layer to improve the optical absorption. Among a variety of such nanophotonic structures designed to improve optical absorption in TFSCs, one-dimensional (1D) periodic grating is one of the most popular approach because it is simple and easy to fabricate. For 1D grating, the waveguide mode resonances or leaky-mode resonances are excited at several wavelengths. Thus the enhancement of absorptance is located near these wavelengths. Consequently, the sharpness of the spectral absorption is the main obstacle for practical application. Considering of this limitation, a novel grating design approach named complex grating was proposed to broaden the spectral absorption. This design is based on the idea of a super-fine structure. The super-fine structure has more complicated structure within the same period comparing to the conventional simple structure. In other words, the complex grating is the superposition of multiple simple gratings with different grating depths in a sub-period, which is the part of the whole period that is corresponding to the conventional simple grating. With the rapid development of micro-manufacturing technology, it is possible to fabricate such nanophotonic structures.

In this paper, we focus on the light trapping in hydrogenated amorphous silicon (a-Si:H) TFSCs because the material a-Si:H possesses the characteristic advantages of: low cost, nontoxicity, abundance, and is convenient to process [17, $\underline{18}]$. A simple trapezoid 
grating structure and complex trapezoid grating structure were investigated by numerical simulation of the spectral absorption utilizing the rigorous coupled-wave analysis. The effects of the geometric parameters on the absorption of the simple grating and complex grating were investigated. Based on the simulated results, the power conversion efficiency $\eta$ of the proposed nanophotonic structure was theoretically analyzed for comparison. The angular response of the complex grating structure was also discussed.

\section{Solar cell structure and simulation method}

The proposed structure of solar cells is schematically shown in Fig. 1. The diffusion length of the short minority carrier in a-Si:H is only about $300 \mathrm{~nm}$. Considering its poor carrier transport, the total thickness $d$ of the active layer is fixed as $300 \mathrm{~nm}$. It should be emphasized that the simple trapezoid grating and the complex trapezoid grating share the same period. For the complex trapezoid grating, one period is divided into three parts, each part has the same sub-period. For each sub-period, it has the same fill ratio $F$ but different grating depth $h$. The process of the parameters optimization is discussed in the following section.

For periodic nanophotonic structure with such small period comparable to the wavelength, it is necessary to take into account the wave effects by solving the full wave vector Maxwell's equations. The rigorous coupled wave analysis (RCWA) method, which is also called the Fourier model method, has been proven to be a rigorous solution of Maxwell's equations and well suited to calculate the reflectance, transmittance and absorption spectra of periodic structures [19-21]. This method fully takes into account the dispersion characteristics of the materials. By using conservation of energy, the absorption of the solar cells can be expressed as:

$$
A(\lambda)=1-\left(\sum_{i} R_{i}+\sum_{i} T_{i}\right)
$$

where $A(\lambda)$ is the wavelength-dependent absorption, $R_{i}$ and $T_{i}$ are the reflected and transmitted efficiencies in the $i$-th diffraction order. The incident wavelength range is
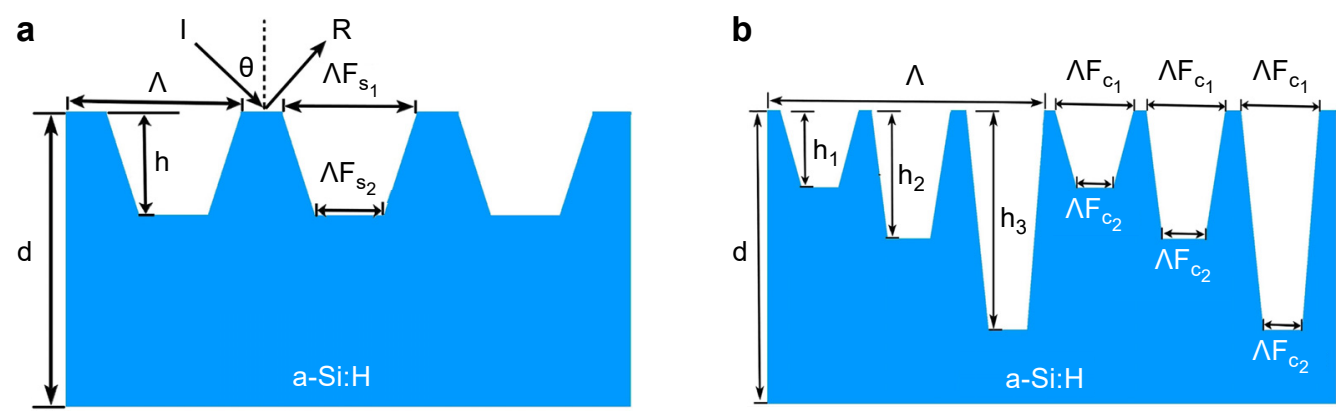

Fig. 1. Schematic of light trapping structure for improving the absorption in thin-film solar cells: the simple trapezoid grating structure (a), and the complex trapezoid grating structure (b). 
from 400 to $800 \mathrm{~nm}$, corresponding to the band gap of a-Si:H. In the simulation, the material parameters of a-Si:H are referenced from PALIK [22].

In order to quantitatively illustrate the absorption performance of solar cells, the power conversion efficiency $\eta$ of solar cells is calculated and compared by the following expression:

$$
\eta=\frac{J_{\mathrm{sc}} V_{\mathrm{oc}} \mathrm{FF}}{P_{\mathrm{in}}}
$$

where $J_{\mathrm{sc}}$ is the short-circuit current density that relates to the photon absorption, $V_{\mathrm{oc}}$ is the open-circuit voltage, $\mathrm{FF}$ is the fill-factor, and $P_{\text {in }}$ is the incident solar power. We assume that one photon can generate an electron-hole pair and all the generated electron-hole pairs can be effectively extracted. The $J_{\text {sc }}$ can be calculated by:

$$
J_{\mathrm{sc}}=\int_{\lambda_{\min }}^{\lambda_{\max }} e \frac{\lambda}{h c} A(\lambda) I_{\mathrm{AM} 1.5}(\lambda) \mathrm{d} \lambda
$$

where $c$ is the speed of light in vacuum, $h$ is the Planck constant, $A(\lambda)$ is the calculated optical absorption spectrum, $I_{\mathrm{AM} 1.5}(\lambda)$ is the incident solar radiation intensity under the standard air mass, namely AM1.5 solar spectrum. The $V_{\mathrm{oc}}$ is estimated by:

$$
V_{\text {oc }}=\frac{k T}{e} \ln \left(\frac{J_{\mathrm{sc}}}{J_{0}}+1\right)
$$

where the value of $k$ is the Boltzmann constant, $T$ is the temperature and the value is $300 \mathrm{~K}, J_{0}$ is the saturation current density and the value is $1.5 \times 10^{-15} \mathrm{~A} / \mathrm{cm}^{2}[\underline{23}]$. The FF can be expressed by the empirical formula:

$$
\mathrm{FF}=\frac{V_{\mathrm{oc}}-\frac{k T}{e} \ln \left(\frac{e V_{\mathrm{oc}}}{k T}+0.72\right)}{V_{\mathrm{oc}}+\frac{k T}{e}}
$$

\section{Results and discussion}

\subsection{Effect of the geometric parameters on the absorption}

For 1D patterned structure, the effect of TM-polarized light is remarkable. The reason of optical absorption is investigated only for TM-polarized light for simplicity. For optimizing the geometric parameters of the simple grating structure, the initial parameters are selected as $\Lambda=390 \mathrm{~nm}, h=100 \mathrm{~nm}, F_{s_{1}}=0.5$, and $F_{s_{2}}=0.25$. The value of the fill ratio $F_{s_{1}} / F_{s_{2}}$ is fixed at 2 . It should be noted that the scanned parameters vary while the other parameters are kept constant. Figure 2 shows the contour plots of the spectral absorption properties as a function of the wavelength for the simple grating structures. 

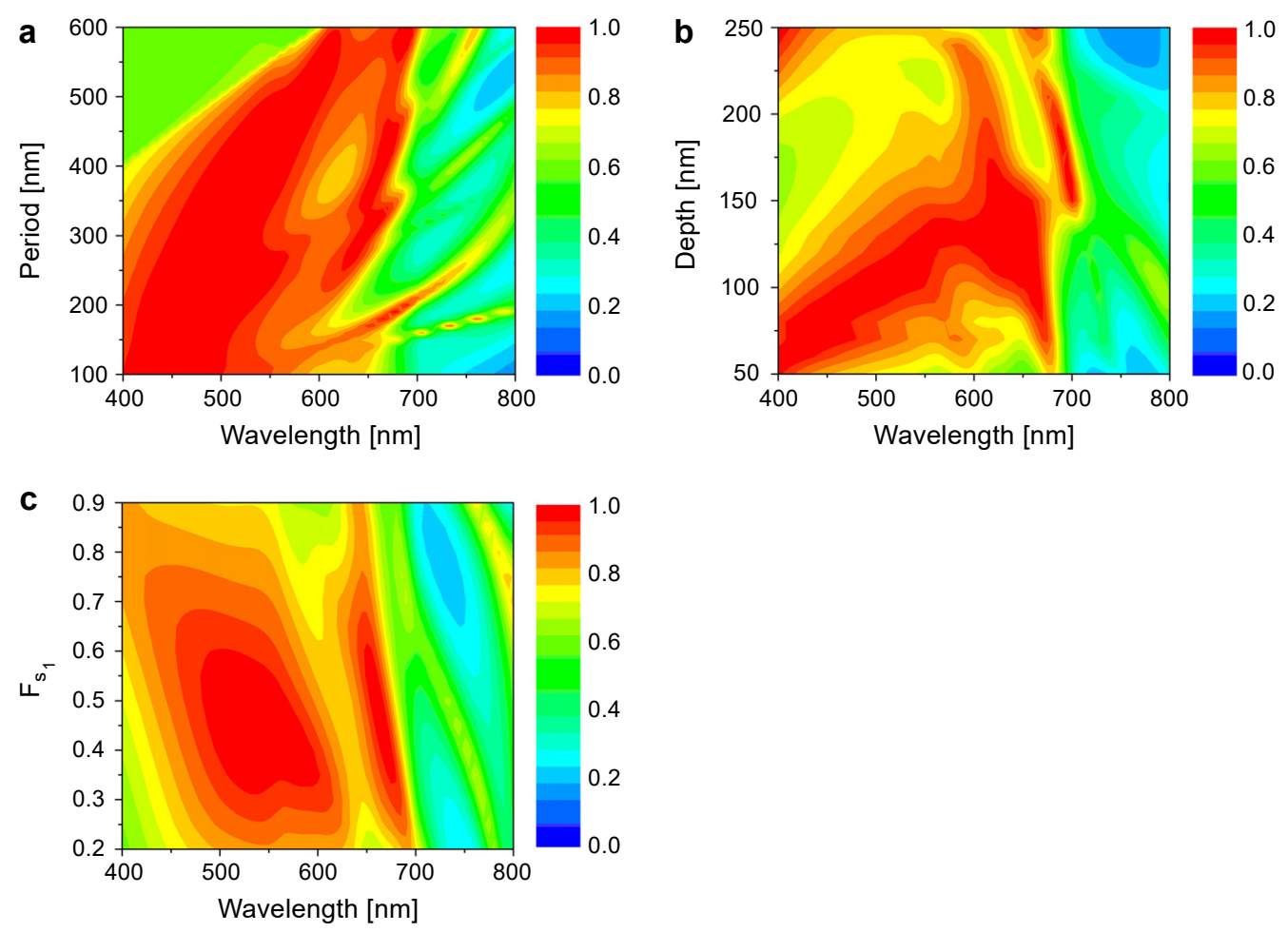

Fig. 2. Contour plots of the spectral absorption properties for the simple grating structure: period (a), depth (b), and fill ratio $F_{s_{1}}(\mathbf{c})$.

It is clear that the large optical absorption usually occurs in the short wavelength range. As seen in Fig. 2a, the absorption decreases with the increment of the period in the short wavelength region, which indicates that smaller period can suppress the reflection in the short wavelength region. As the period increases, the high absorption can shift to the long wavelength range due to exciting the resonance effects. It is well known that the simple trapezoid grating structure can excite the waveguide mode resonances, leading to light trapping and to an increase in the absorption in the active layer [1] $]$. Meanwhile, these resonance behaviors strongly depend on the geometric parameters. Thus, the depth and the fill ratio possess the optimal value to make the absorption maximum, which can be shown in Figs. $2 \mathbf{b}$ and $2 \mathbf{c}$.

In order to illustrate the advantages of the super-fine structure, the period of the complex trapezoid grating is equal to that of the simple trapezoid grating and is fixed as $390 \mathrm{~nm}$ according to the optimization results of the simple grating. The ratio of the fill ratio $F_{c_{1}} / F_{c_{2}}$ is kept as 2 as well. The ratio of the different depths $h_{1}: h_{2}: h_{3}$ is selected as $1: 2: 3$. The effect of the fill ratio and the depths on the absorption of the complex trapezoid grating is shown in Fig. 3. As shown in Fig. 3a, it is clear that the absorption with the fill ratio $F_{c_{1}}=0.8$ is larger than that with the $F_{c_{1}}=0.5$. When the fill ratio 

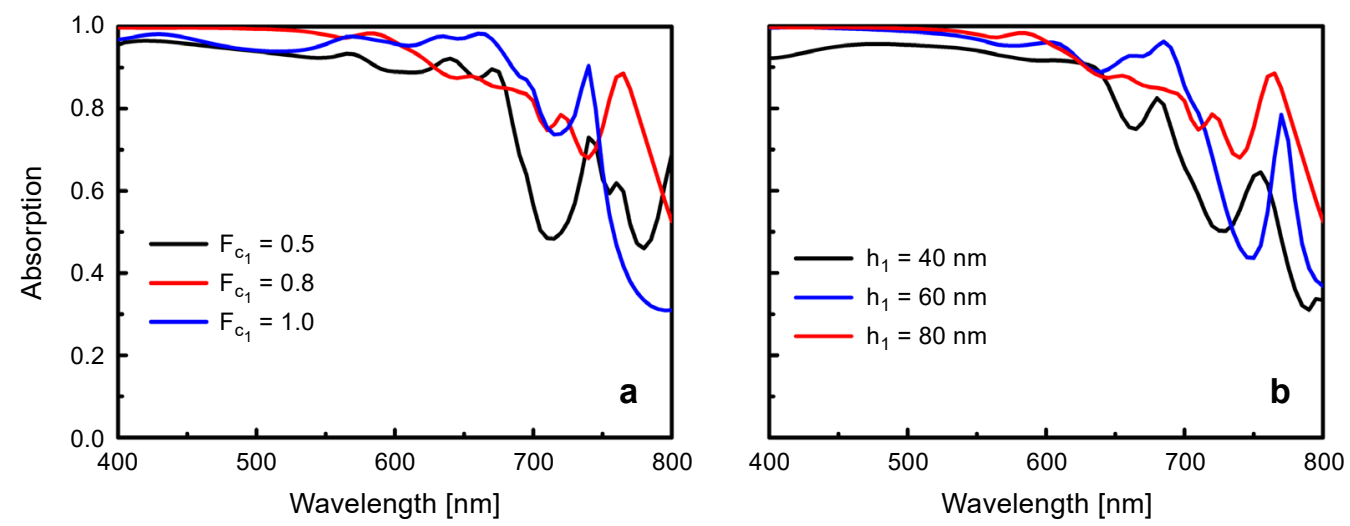

Fig. 3. Effect of the fill ratio and depth on the spectral absorption of the complex trapezoid grating structure: fill ratio (a), and depth (b).

further increases to $F_{c_{1}}=1$, the absorption in the short wavelength region decreases. As seen in Fig. 3b, the spectral absorption is significantly increased as the depth increases. Obviously, the spectral absorption with depth $h_{1}=80 \mathrm{~nm}$ exhibits the best performance.

\subsection{Comparison of simple grating structure and complex grating structure}

Figure 4 shows the optical absorption of the proposed nanophotonic structure. For comparison, the absorption spectra of the planar a-Si:H and with silica antireflective coating are also plotted in this figure. The thickness of the antireflective coating $\mathrm{SiO}_{2}$ is $70 \mathrm{~nm}$. Apparently, the optical absorption of the antireflective coating is enhanced compared to the planar structure, revealing effective properties to suppress reflection. To elucidate the light trapping effect, we compared the performance of nanophotonic structure under

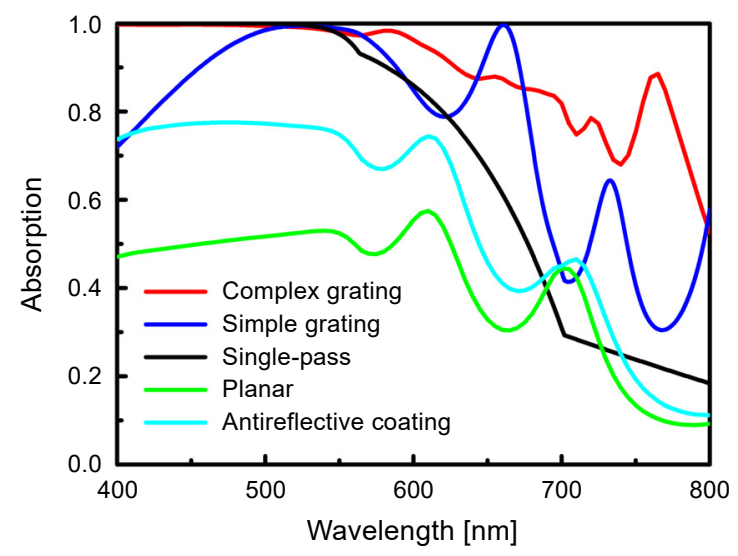

Fig. 4. Comparing the absorption spectra of a planar, antireflective coating, simple trapezoid grating and complex trapezoid grating with the single-pass absorption to show the light trapping effect. 
study to single-pass absorption as well. The single-pass absorption is assuming perfect antireflection but no light trapping [24]. That is, the normal incident light passes through the semiconductor material only once. The absorption of single-pass can be expressed as:

$$
A_{\text {single-pass }}=1-\exp (-\alpha d)
$$

where $\alpha$ is the absorption coefficient, $d$ is the film thickness. As seen in Fig. 3a, the absorption of complex trapezoid grating in short wavelength less than $500 \mathrm{~nm}$ is close to 1 indicating that the perfect antireflective effect is achieved. Whereas the Fresnel reflection loss is still existent for the planar slab and simple trapezoid grating in this wavelength region. This phenomenon can be attributed to the gradual change of the refractive index. As the wavelength increased more than $500 \mathrm{~nm}$ to the long wavelength region, the light trapping effect performs more and more markedly. The physical mechanism of light trapping is expressed as follows. For planar thin film, the light trapping effect is mainly attributed to Fabry-Pérot resonance modes due to the large index contrast between air and a-Si:H layer, which is ubiquitous in the thin film layer [25]. While for simple trapezoid grating, the waveguide mode resonance or leaky-mode resonance effects lead to light trapping and increase the absorption in the active layer [13]. The reason of the light trapping effect for the complex trapezoid grating is analogous to that of simple grating. In addition, the complex trapezoid grating with different depths can excite microcavity resonance effect, which can trap light effectively [26]. Both the excellent antireflective properties and microcavity effect make the complex grating outperform to the simple grating.

In order to quantitatively compare the optical absorption performance with different structures, the $\eta$ the is calculated in the Table. The $J_{\mathrm{sc}}$ is mainly determined by the optical absorption. Also, the $\eta$ of the simple grating with different depths is calculated respectively for comparison. As shown in the Table, it is clear that the complex grating structure holds the highest $J_{\text {sc }}$ compared to other structures, in accordance to the spectral absorption. The $\eta$ of the nanophotonic structure is much more higher than that of the planar structure. The $\eta$ of the complex grating structure is $15.60 \%$, where that is $13.31 \%$ for simple grating structure and $9.49 \%$ for antireflective coating structure. The optimized complex grating structure shows $17.2 \%$ higher $\eta$ than the simple grating struc-

$\mathrm{T}$ a b 1 e. The power conversion efficiency of a-Si:H solar cells with different structures.

\begin{tabular}{llllc}
\hline Structure & $J_{\text {sc }}\left[\mathrm{mA} / \mathrm{cm}^{2}\right]$ & $V_{\text {oc }}[\mathrm{V}]$ & FF & Efficiency \\
\hline Planar & 10.33 & 0.764 & 0.856 & $6.76 \%$ \\
Antireflective coating & 14.35 & 0.772 & 0.857 & $9.49 \%$ \\
Simple grating & 19.87 & 0.781 & 0.858 & $13.31 \%$ \\
Super-fine $h_{1}=h_{2}=h_{3}=80 \mathrm{~nm}$ & 16.47 & 0.776 & 0.857 & $10.95 \%$ \\
Super-fine $h_{1}=h_{2}=h_{3}=160 \mathrm{~nm}$ & 17.08 & 0.777 & 0.858 & $11.39 \%$ \\
Super-fine $h_{1}=h_{2}=h_{3}=240 \mathrm{~nm}$ & 15.99 & 0.777 & 0.858 & $10.66 \%$ \\
Complex grating $h_{1}=80 \mathrm{~nm}, h_{2}=160 \mathrm{~nm}, h_{3}=240 \mathrm{~nm}$ & 23.14 & 0.785 & 0.859 & $15.60 \%$ \\
\hline
\end{tabular}



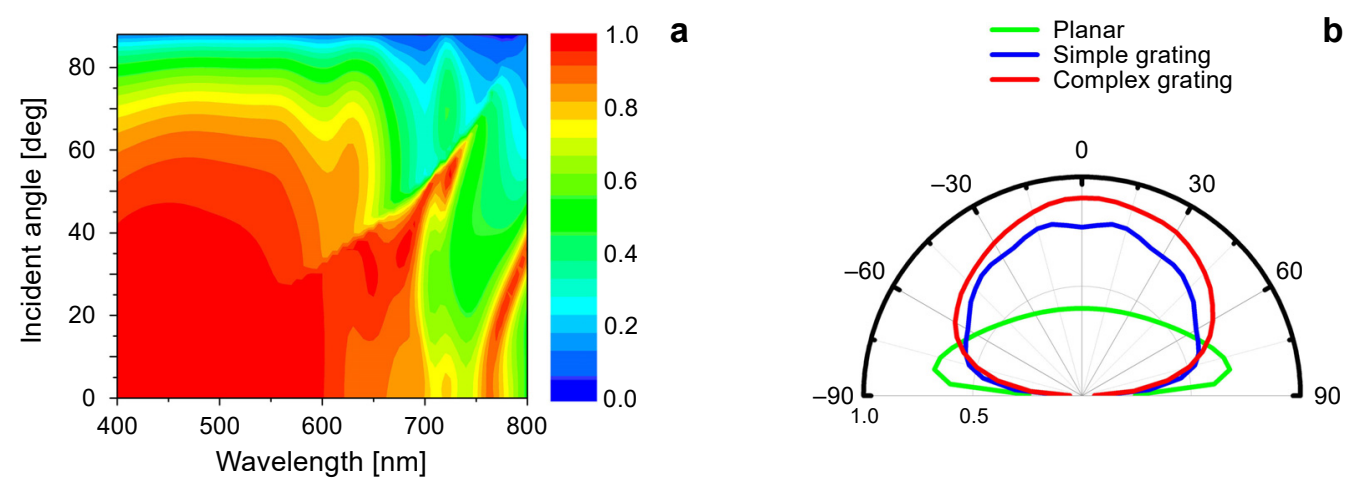

Fig. 5. Contour plots of the spectral absorption properties for the complex grating structure as the function of the incident angle (a). The incident-angle-dependence of the averaged absorption of a planar, simple trapezoid grating and complex trapezoid grating for TM polarized light (b).

ture, indicating that super-fine structure with different depths is the priority selection for TFSCs. The $\eta$ of the super-fine simple grating structure with the same depth is lower than the simple grating structure, which means that the optical absorption of the simple structure outperforms to the super-fine simple grating.

\subsection{Effect of the incident angle}

As sunlight impinges on the surface of solar cells from every direction, the angular influence is of crucial importance, so the angular response of the solar cell is also examined. Figure $5 \mathbf{a}$ shows the contour plots of the spectral absorption properties for the complex grating structure as the function of an incident angle. The spectral absorption almost remains unchanged in the short wavelength region when the incident angle is lower than 60 degree, exhibiting the excellent omnidirectional characteristics. The integrated absorption over all possible input angles is the best way to show the performance of solar cells. Figure $5 \mathbf{b}$ represents the wavelength integrated polar plot to make the angular dependence outstanding. It is clear that the complex grating provides the best performance at almost all angles compared to the simple grating.

\section{Conclusion}

In summary, a novel light trapping structure composed of a complex trapezoid grating is proposed to effectively improve the optical absorption of the a-Si:H TFSCs. The optical absorption properties are obtained by using the RCWA numerical method. The calculating results indicate that the optimized simple trapezoid grating shows $97 \%$ enhancement of the power conversion efficiency while the complex trapezoid grating shows $131 \%$ enhancement. It is clear that the complex trapezoid grating outperforms the simple grating in enhancing light absorption in the silicon-based TFSCs, which is attributed to the perfect antireflective effects and microcavity resonance. In addition, the angular response of the optical absorption is also investigated for the a-Si:H TFSCs 
and the results show that the complex grating exhibits excellent omnidirectional properties. Therefore, such broadband and omnidirectional properties of the proposed super-fine structure can be widely used in a thin film photovoltaic field, such as gallium arsenide (GaAs), cadmium telluride (CdTe), copper indium gallium selenide (CIGS), perovskite solar cells and so on.

Acknowledgments - This work was supported by the Natural Science Foundation of Shaanxi Province (No. 2019JM-508), the Scientific Research Program Funded by Shaanxi Provincial Education Department (No. 19JK0186) and the Scientific Research Project of Shaanxi University of Technology (No. SLGQD1815).

\section{References}

[1] Luque A., Hegedus S., Handbook of Photovoltalic Science and Engineering, Wiley Press, England, 2003.

[2] Fahr S., Ulbrich C., Kirchartz T., Rau U., Rockstuhl C., Lederer F., Rugate filter for light-trapping in solar cells, Optics Express 16(13), 2008, pp. 9332-9343, DOI: 10.1364/OE.16.009332.

[3] Atwater H.A., Polman A., Plasmonics for improved photovoltaic devices, Nature Materials 9(3), 2010, pp. 205-213, DOI: $10.1038 /$ nmat2629.

[4] Акімоv Yu.A., Кон W.S., Design of plasmonic nanoparticles for efficient subwavelength light trapping in thin-film solar cells, Plasmonics 6(1), 2011, pp. 155-161, DOI: 10.1007/s11468-010-9181-4.

[5] Акімоv Yu.A., Кон W.S., Ostrikov K., Enhancement of optical absorption in thin-film solar cells through the excitation of higher-order nanoparticles plamon modes, Optics Express 17(12), 2009, pp. 10195-10205, DOI: 10.1364/OE.17.010195.

[6] Temple T.L., Mahanama G.D.K., Reehal H.S., Bagnall D.M., Influence oflocalized surface plasmon excitation in silver nanoparticles on the performance of silicon solar cells, Solar Energy Materials and Solar Cells 93(11), 2009, pp. 1978-1985, DOI: 10.1016/j.solmat.2009.07.014.

[7] Qiao L., Wang D., Zuo L., Ye Y., Qian J., Chen H., He S., Localized surface plasmon resonance enhanced organic solar cell with gold nanospheres, Applied Energy 88(3), 2011, pp. 848-852, DOI: 10.1016/j.apenergy.2010.09.021.

[8] Ferry V.E., Sweatlock L.A., Pacifici D., Atwater H.A., Plasmonic nanostructure design for efficient light coupling into solar cells, Nano Letters 8(12), 2008, pp. 4391-4397, DOI: 10.1021/n18022548.

[9] Abass A., Shen H., Bienstman P., Maes B., Angle insensitive enhancement of organic solar cells using metallic grating, Journal of Applied Physics 109(2), 2011, article 023111, DOI: 10.1063/1.3533980.

[10] Pala R.A., White J., Barnard E., Liu J., Brongersma M.L., Design of plasmonic thin-film solar cells with broadband absorption enhancements, Advanced Materials 21(34), 2009, pp. 3504-3509, DOI: $10.1002 /$ adma.200900331.

[11] Shen H., MAes B., Combined plasmonic gratings in organic solar cells, Optics Express 19(S6), 2011, pp. A1202-A1210, DOI: 10.1364/OE.19.0A1202.

[12] Fei Guo C., Sun T., CAo F., Liu Q., Ren Z., Metallic nanostructures for light trapping in energy-harvesting devices, Light: Science and Applications 3(4), 2014, article e161, DOI: 10.1038/1sa.2014.42.

[13] Wu W., Magnusson R., Total absorption of TM polarized light in a $100 \mathrm{~nm}$ spectral band in a nanopatterned thin a-Si film, Optics Letters 37(11), 2012, pp. 2103-2105, DOI: 10.1364/OL.37.002103.

[14] Martins E.R., Li J., Liu Y.K., Zhou J., Krauss T.F., Engineering gratings for light trapping in photovoltaics: the supercell concept, Physical Review B 86(4), 2012, article 041404(R), DOI: 10.1103/PhysRevB.86.041404.

[15] Deng J., Wang M., Yang Z., Liu J., Sun Z., Song X., Application of patterned growth of aligned zinc oxide nanoarrays by mirocontact printing in quantum dots-sensitized solar cells, Journal of Power Sources 280, 2015, pp. 555-564, DOI: 10.1016/j.jpowsour.2015.01.137. 
[16] Deng J., Wang M., Yang Z., Yang Y., Zhang P., Preparation of TiO 2 nanoparticles two-dimensional photonic-crystals: a novel scattering layer of quantum dot-sensitized solar cells, Materials Letters 183, 2016, pp. 307-310, DOI: 10.1016/j.matlet.2016.07.139.

[17] Zhu J., Yu Z., Burkhard G.F., Hsu C.M., Connor S.T., Xu Y., Wang Q., McGehee M., Fan S., CuI Y., Optical absorption enhancement in amorphous silicon nanowire and nanocone arrays, Nano Letters 9(1), 2009, pp. 279-282, DOI: 10.1021/n1802886y.

[18] Wang W., Wu S., Reinhardt K., Lu Y., Chen S., Broadband light absorption enhanccment in thin -film silicon solar cells, Nano Letters 10(6), 2010, pp. 2012-2018, DOI: 10.1021/n1904057p.

[19] Moharam M.G., GaYlord T.K., Diffraction analysis of dielectric surface-relief gratings, Journal of the Optical Society of America 72(10), 1982, pp. 1385-1392, DOI: 10.1364/JOSA.72.001385.

[20] Moharam M.G., GAYlord T.K., Rigorous coupled-wave analysis of metallic surface-relief gratings, Journal of the Optical Society of America A 3(11), 1986, pp. 1780-1787, DOI: 10.1364/JOSAA.3. $\underline{001780}$.

[21] Moharam M.G., Grann E.B., Pommet D.A., Gaylord T.K., Formulation for stable and efficient implementation of the rigorous coupled-wave analysis of binary gratings, Journal of the Optical Society of America A 12(5), 1995, pp. 1068-1076, DOI: 10.1364/JOSAA.12.001068.

[22] Palik E.D., Handbook of Optical Constants of Solids, Academic Press, San Diego, 1985.

[23] Feng N., Michel J., Zeng L., Liu J., Hong C., Kimerling L.C., Duan X., Design of highly efficient light-trapping structures for thin-film crystalline silicon solar cells, IEEE Transactions on Electron Devices 54(8), 2007, pp. 1926-1933, DOI: 10.1109/TED.2007.900976.

[24] Wang K.X., Yu Z., Liu V., Cui Y., Fan S., Absorption enhancement in ultrathin crystalline silicon solar cells with antireflection and light-trapping nanocone gratings, Nano Letters 12(3), 2012, pp. 1616-1619, DOI: 10.1021/nl204550q.

[25] Meng X., Depauw V., Gomard G., El Daif O., Trompoukis C., Drouard E., Jamois C., Fave A., Dross F., Gordon I., Seassal C., Design, fabrication and optical characterization of photonic crystal assisted thin film monocrystalline-silicon solar cells, Optics Express 20(S4), 2012, pp. A465 -A475, DOI: 10.1364/OE.20.00A465.

[26] Cheng Q., Li P., Lu J., Yu X., Zhou H., Silicon complex grating with different groove depths as an absorber for solar cells, Journal of Quantitative Spectroscopy and Radiative Transfer 132(2), 2014, pp. 70-79, DOI: 10.1016/j.jqsrt.2013.01.027. 